\title{
A Case of Aortic Dissection Determined with Clinical Suspicion in Young Female Patient Who Admitted with Chest and Back Pain
}

\author{
Aksoy $\mathrm{I}^{* 1}$, Duran $\mathrm{L}^{1}$, Yamanlar $\mathrm{OL}^{2}$, Gurler $\mathrm{S}^{1}$, Senguldur $\mathrm{E}^{1}$ and Deser $\mathrm{SB}^{3}$ \\ ${ }^{1}$ Ondokuz Mayıs University Emergency Medicine, Samsun, Turkey \\ ${ }^{2}$ Mehmet Aydin Education and Research Hospital, Samsun, Turkey \\ ${ }^{3}$ Ondokuz Mayıs University Cardiovascular Surgery, Samsun, Turkey
}

*Corresponding author: Aksoy I, Department of Emergency Medicine, Faculty of Medicine, Ondokuz Mayis University, Samsun, Turkey, 55100, Tel: +905458961555, E-mail: driskenderaksoy@hotmail.com, iskender.aksoy@ omu.edu.tr

Citation: Aksoy I, Duran L, Yamanlar OL, Gurler S, Senguldur E, et al. (2017) A Case of Aortic Dissection Determined with Clinical Suspicion in Young Female Patient Who Admitted with Chest and Back Pain. SAJ Case Rep 4: 402

Article history: Received: 28 September 2017, Accepted: 28 November 2017, Published: 30 November 2017

\begin{abstract}
Aortic dissection is an urgent clinical situation and mortality is not have been diagnosed early that presenting with usually chest and back pain and seen more frequently in men and over 50 years. We aimed to present a young women patient who admitted to emergency service with chest and back pain and had diagnoses of the aortic dissection although negative initial diagnostic tests and had continued clinical suspicion.

A 37-year-old female patient presented with a chest pain that was reflected in the back. No pathology was found in the examinations performed in other hospitals. Blood tests, electrocardiography, echocardiography and contrast-enhanced computed tomography are normal. Aortic dissection is detected in a contrast-enhanced computerized tomography, which is taken again to the patient after the pain does not pass.

Clinical suspicion is very important for the diagnosis of aortic dissection. The patient could not be diagnosed by repeated examination and the patient was diagnosed with the second tomography taken on suspicion of clinical suspicion. Patients should not be discharged unless the clinical suspicion is terminated.
\end{abstract}

Keywords: Aortic dissection; Debekay type 2; Chest pain

\section{Introduction}

Aortic dissection (AD) is one of the real cardiovascular emergencies; defined as the separation of media layer of aorta from the intima layer along its' long axis [1]. It often presents severe chest pain and acute hemodynamic compromise. The mortality of aortic dissection remains at 25 to 30 percent. Early and accurate diagnosis and treatment are crucial for survival. While it can be seen in young individuals with soft tissue disease, the real peak is seen after the age of 50 [2]. It is seen two times more in males than females [3]. In this case, we wanted to emphasize that the diagnosis of aortic dissection was established with second computed tomographic (CT) angiography which was taken after 10 hours because of the continued clinical suspicion, in a young female patient whom referred to emergency department with chest and back pain and could not get the diagnosis of aortic dissection with the CT angiography and echocardiography (ECHO).

\section{Case Report}

37 year old female patient admitted to our emergency department with belt-like pain existing by 24 hours which started suddenly below the left mammary and radiated to back. There was no concomitant nausea, vomiting, breath shortness. There were no any positive findings in tests performed in other clinic which she applied with the complaint of pain. She was given analgesic medication in district hospital after Miyocardial Infarction (MI) and pneumotorax eliminated. Also intramuscular steroid injection was performed by her orthopedist uncle to the region of back pain for thoracic nerve blockade. The patient was discharged after partial decrease of the pain. The patient admitted to another clinic because of relapsing of the pain and she was referred to 
educational research hospital to investigate with CT. Complete blood count, cardiac enzymes and limited biochemical tests were found normal. Non-contrast CT was taken because the other clinic physicians did not think pulmonary thromboembolic or aortic dissection. Non-contrast CT scan revealed no pathologic findings and the patient was given narcotic analgesic medication again and discharged after improvement of the pain. Patient admitted to our emergency department two hours after discharge from the prior hospital due to the belt-like pain which started again below the left mammary and radiating to the back.

No disease except hypothyroidism and no drug usage other than levothyroxine $200 \mathrm{mcg}$ /day were found in patient's medical history. There is no known connective tissue disease in the patient and there was no evidence to think Marfan syndrome. In her family history, no cardiac disease was reported.

Physical examination was normal and at the time of admission. All vital signs were stable.

Electrocardiography was compatible with normal sinus rhythm (Figure 1). In complete blood count; Leukocyte: 9000/ $\mu \mathrm{L}$, Hemoglobin (Hbg): 13g/dL, Platelet (Plt): 304000/ $\mu \mathrm{L}$; biochemical parameters (sodium, potassium, calcium, creatinine, Aspartat Transaminaz (AST), Alanine Aminotransferase (ALT), amylase, lipase) and troponin-I were found normal. In venous blood gas; pH: 7.50, pCO2: $27 \mathrm{mmHg}, \mathrm{HCO} 3: 22 \mathrm{mmol} / \mathrm{L}$ and fingertip oxygen saturation was 98\%. D-dimer was found as $625 \mathrm{ng} / \mathrm{mL}$ (upper limit is 500). Thorax CT (Toshiba Active, 2011, Japan) scan with embolism protocol and contrast-enhanced abdominal CT angiography with pre-diagnoses of aortic dissection and pulmonary thrombo embolism (PTE) were performed. Radiologic evaluation indicated that no filling defect compatible with pulmonary embolism was seen; however ascending aorta was approximately $27 \mathrm{~mm}$, descending aorta was $23 \mathrm{~mm}$ and there was a suspicious area in ascending aorta having a diameter of $1 \mathrm{~cm}$ (Figure 2). No organ pathology, fluid, mass etc. were found in abdominal CT scan. In the ascending aorta, it is stated that there may be artefacts for the region where the dissection is thought.

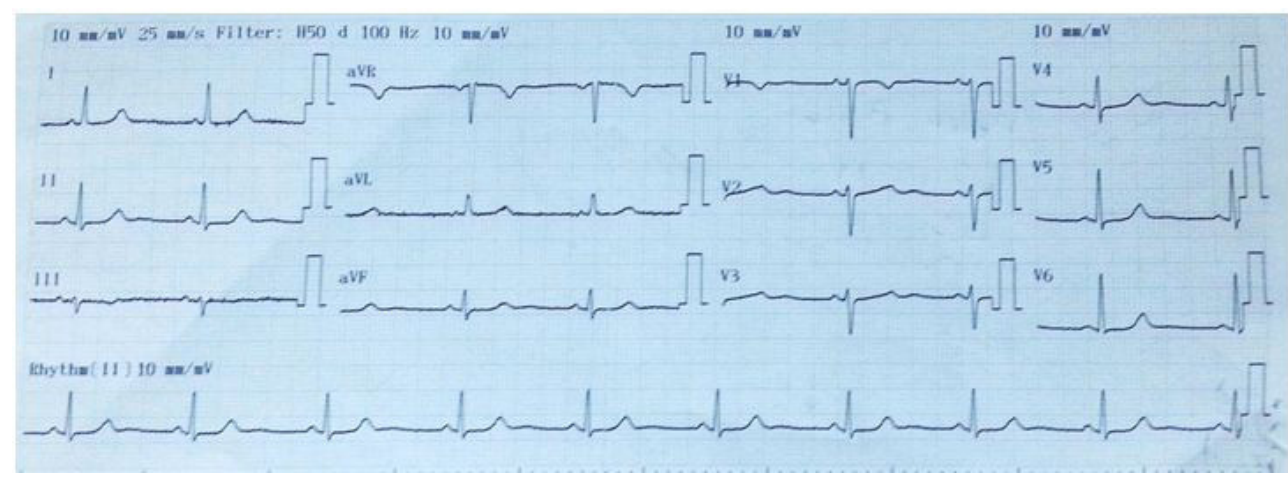

Figure 1: Patient's ECG

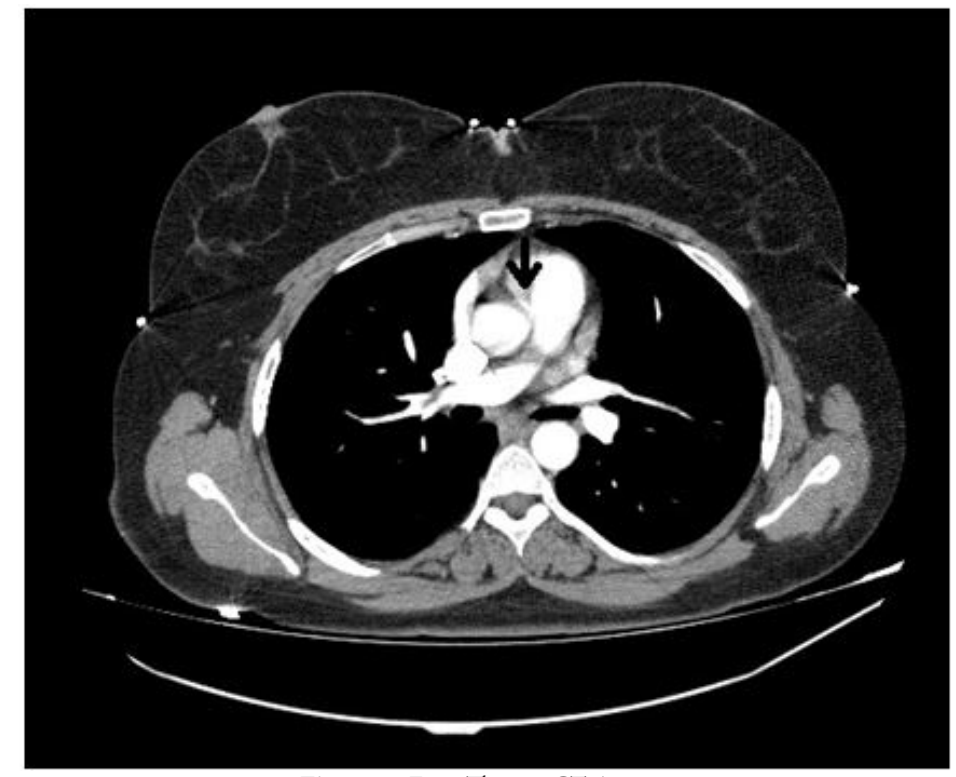

Figure 2: First Thorax CT Anjio

Patient was consulted to pulmonology department with the suspicion of PTE because of high D-dimer value, unexplained chest and back pain, hypocarbia in blood gas. Due to the diagnosis of PTE couldn't rule out, pulmonologist suggested some further tests (ECHO, bilateral lower extremity venous doppler ultrasonography, ventilation/perfusion scintigraphy) and low molecular weight heparin treatment after ruling out aortic dissection. In addition, the patient was consulted to cardiovascular surgery department with the pre-diagnosis of aortic dissection. To assessment made by cardiovascular surgeon, diagnoses of aortic dissection couldn't rule out. They suggested ECHO or control contrast-enhanced abdominal CT angiography 6-10 hours later. In the evaluation with 
ECHO, pulmonary artery pressure was $32 \mathrm{mmHg}$, ejection fraction was 66\%, left ventricule diameters and wall movements were normal, aortic valves were naturally and there was no dissection flap. Patient was reevaluated with ECHO report by cardiovascular surgeon and because of the suspicion of dissection still continued, contrast-enhanced thorax CT angiography was performed again, after controlled serum creatinine value was normal. Thorax CT angiography was compatible with acute Debakey type 2 aortic dissection starting from ascending aorta (Figure 3). Patient was operated immediately by cardiovascular surgeon with the diagnosis of acute Debakey type 2 aortic dissections. Ascending aorta was excised $1 \mathrm{~cm}$ distal. The origin of the dissection was detected in the posterior wall of the ascending aorta. With a successful operation, our patient stayed in critical care for 2 days and on the third day, the patient could be feeded orally. The patient was discharged as healthy.

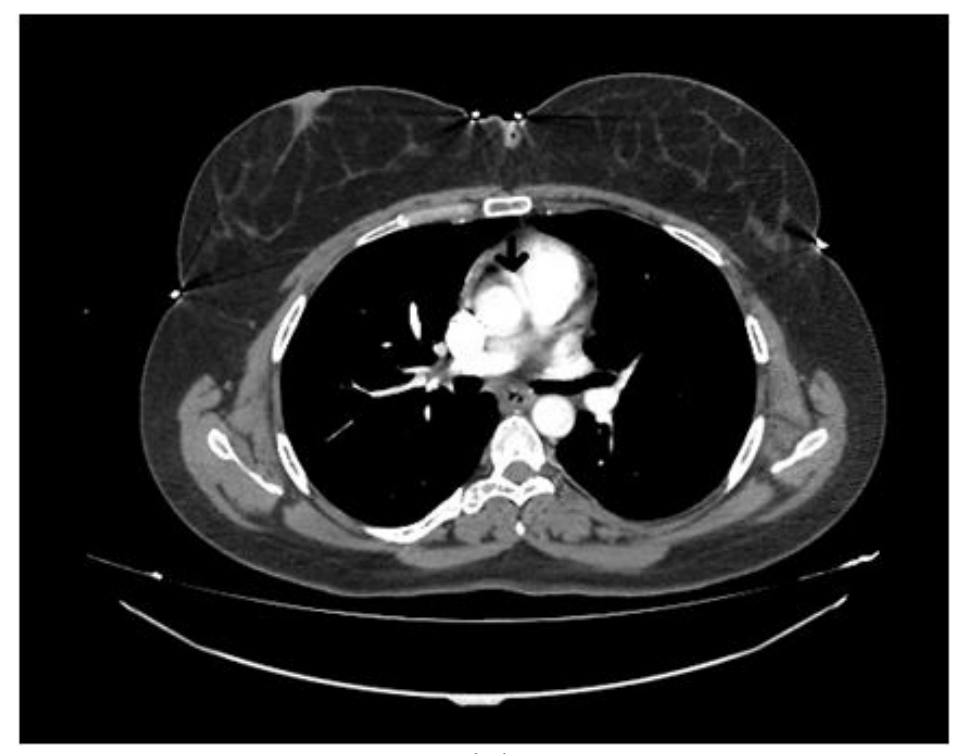

Figure 3: Second Thorax CT Anjio

\section{Conclusion}

The most common symptoms of aortic dissection which is one of the important patterns in cardiovascular emergencies, are sudden and tearing chest and back pain [3]. Except having a congenital heart disease, family history and pregnancy it is rarely seen under age of 40 [4]. The mortality of untreated cases with ascending aortic dissection increases in hours [5].

Ascending aortic dissections are almost twice as common as descending dissections. The right lateral wall of the ascending aorta is the most common site of aortic dissection [6]. In patients with an ascending aortic dissection, aortic arch involvement occurs in up to $30 \%$ [7].

Aortic dissection is rare in young woman without connective tissue disease or Marfan syndrome. In this case, patient was referred to our emergency department 24 hours after the complaints of sudden chest and back pain started and could not be diagnosed with CT scans and ECHO. Patient referred three hospitals until referred to our emergency department and none of them thought aortic dissection. Patient whom tested many times by our department and other clinics and could not be diagnosed, followed up insistently and diagnosed with the second CT angiography and treated with a successful operation before without mortality.

As a conclusion, clinical suspicion is very important for the diagnosis of aortic dissection. In this case, we wanted to emphasize that following up the patients must be continued until the end of the clinical suspicion.

\section{References}

1. Mukherjee D, Eagle KA (2005) Aortic dissection--an update. Curr Probl Cardiol 6: 287-325.

2. Eskandari MK, Resnick SA (2005) Aneurysms of the renal artery. Semin Vasc Surg 4: 202-8.

3. Donovan EM, Seidel GK, Cohen A (2000) Painless aortic dissection presenting as high paraplegia: A case report. Arch Phys Med Rehabil 10: 1436-8.

4. DeBakey ME, McCollum CH, Crawford ES, Morris GC, Howell J, et al. (1982) Dissection and dissecting aneurysms of the aorta: twenty-year follow-up of five hundred twenty-seven patients treated surgically. Surg 6: 1118-34.

5. Dettmeyer R, Schmidt P, Madea B (1998) Two cases of unexpected sudden death due to cystic medionecrosis of the aorta associated with bloodless aortic dissection. Forensic Sci Int 3: 161-6.

6. Larson EW, Edwards WD (1984) Risk factors for aortic dissection: a necropsy study of 161 cases. Am J Cardiol 6: 849-55.

7. Nienaber CA, Eagle KA (2003) Aortic dissection: new frontiers in diagnosis and management: Part II: therapeutic management and follow-up. Circulation 6: $772-8$. 